Review Article

\title{
Bezold Jarisch Reflex- New Interest, Old Phenomenon
}

\author{
Sanjeev Arya ${ }^{1,}$, Shantanu Belwal ${ }^{1}$, Bhupesh Uniyal ${ }^{1}$, Bhuwan Tiwari ${ }^{3}$, Preeti Sharma ${ }^{2}$ \\ ${ }^{1}$ Medical Intensive Care Unit, Max Super Specialty Hospital, Dehradun, India \\ ${ }^{2}$ Department of Cardiology, Max Super Specialty Hospital, Dehradun, India \\ ${ }^{3}$ Department of Cardiology, Ram Manohar Lohia Institute of Medical Sciences, Gomti Nagar, Lucknow, India
}

Email address:

Sanjeev.arya@hotmail.co.uk (S. Arya)

${ }^{*}$ Corresponding author

\section{To cite this article:}

Sanjeev Arya, Shantanu Belwal, Bhupesh Uniyal, Bhuwan Tiwari, Preeti Sharma. Bezold Jarisch Reflex- New Interest, Old Phenomenon. American Journal of Internal Medicine. Vol. 8, No. 1, 2020, pp. 24-29. doi: 10.11648/j.ajim.20200801.15

Received: December 13, 2019; Accepted: January 6, 2020; Published: January 16, 2020

\begin{abstract}
Bezold Jarisch reflex (BJR) is an eponym for a triad of responses namely: bradycardia, hypotension and vasodilation that develops upon stimulation of intracardiac mechanoreceptors and is mediated by vagal afferent nerve fibers. Though it was first reported in 1867 it remains a topic of discussion due to its implications in various clinical scenarios. The reflex can be stimulated either by mechanical or chemical stimulators. Often considered a physiologic response, if identified by the clinician at earlier phase, can avoid cardiac complication, it also acts as a prognostic indicator in various diseases. This review focuses on the physiology of the BJR and its possible physiologic role in several clinical situations. We used evidence obtained from a computerized literature of medical databases search using PubMed/Medline/NIH (all articles till October 2019). The search was carried out with MeSH phrase "Bezold Jarisch reflex" and articles were limited to English language only. The relevant published reports, abstracts and human studies with clinical cases to determine the clinical implications of BJR were considered for the review. It has been concluded that BJR is a physiologic response that can occur in number of clinical scenarios. Therefore, simultaneous occurrence of bradycardia and hypotension should alert clinicians to the possibility of BJR.
\end{abstract}

Keywords: Bezold Jarisch Reflex, C-fibers, Chemoreceptors, Myocardial Receptors

\section{Introduction}

Cardiopulmonary reflexes belong to the autonomic or involuntary nervous system. Of which Bezold Jarisch reflex (BJR) is an inhibitory reflex induced by the stimulation of receptors located in the epicardium of the infero-posterior region of the left ventricle.

Stimulation of these 'inhibitory cardiac receptors' by stretch, chemical substances or drugs increases parasympathetic activity and inhibits sympathetic activity.

BJR is interrupted by cervical vagotomy which indicates that the parasympathetic innervation of the cardiopulmonary organs is essential in the transmission of the afferent and/or efferent groups of nerve impulses.

In vasoplegic states, peripheral venous blood pooling may lead to hyper stimulation of the myocardium paradoxically resulting in reflex cardiovascular depression with vasodilation, hypotension and bradycardia which in turn modulates renin release and vasopressin secretion [1-3].

Upon administration of iv veratrum alkaloids in experimental animals von Bezold and Hirt in 1867 first observed the profound decrease in blood pressure and heart rate in conjunction with apnea. The response was eliminated by cutting both cervical part of vagus nerve. Cramer in 1915 described apnea, hypotension, and bradycardia following intravenous injection of veratrum viride extracts in cats and he believed that the receptors for the reflex were in the lung. Later in the late 1930s, Jarisch and Richter reported that the powerful depressor action induced by intravenous veratridine was attributable to the cardiac branches of vagus nerves in cats. They confirmed that the depressor effect initially observed in 1867 by Bezold was reflex in origin whose receptors were located in the ventricle.

With further studies, it has been established that this reflex originates mainly in the cardiopulmonary receptors on the 
unmyelinated and type C-vagal afferent fibers [4, 5]. Dawes et al in 1947 in their study with veratridine in cats showed that the reflex apnea was caused by a mechanism different from that mediating the hemodynamic changes. Subsequent investigators, mostly pharmacologists and physiologists, referred to the triad of responses as Bezold Jarisch reflex or Bezold Jarisch effect, the latter, to emphasize that veratrum alkaloids have multiple actions on several groups of reflexes and also directly on the medullary centers. Today, the BJR connotes the reflexes described by Dawes in the mid twentieth century: bradycardia, vasodilation, and hypotension resulting from stimulation of cardiac receptors $[3,6]$.

Though Bezold Jarisch reflex was discovered long back, it still is a topic of experimental curiosity to the researchers with the anticipation to explore the reflex in as many clinical situations, so as to, identify the complications at an early stage and treat accordingly. This review focuses on the physiology of the BJR and its possible physiologic role in a number of clinical situations.

\section{Material and Methods}

A computerized literature of medical databases search using PubMed/Medline/NIH (all articles till October 2019) was carried out. MeSH phrase used was "Bezold Jarisch reflex". The only inclusion criterion was to review published reports, abstracts and human studies with clinical cases to determine the clinical implications of Bezold Jarisch reflex. The search was limited to English articles only.

\section{Results}

A total of 400 articles appeared in the search results. Upon filtering the articles, a total of 20 complete articles considered relevant to our review were used. In literature, publications mention BJR as a response during physiologic states with emphasis on the clinical implications in disease conditions such as myocardial ischemia and infarction, hemorrhage, hypovolemia, vasovagal syncope, coronary arteriography reperfusion, coronary artery stenosis, Cushing's syndrome, BJR during neurosurgery, craniotomy and spinal surgeries.

\section{Discussion}

Cardiopulmonary reflexes belong to the autonomic or involuntary nervous system and consist of sensory receptors in the heart and lungs, the sensory or afferent fibers in the parasympathetic cranial nerves, medullary centers regulating visceral function by reciprocal activity on parasympathetic and sympathetic innervations, preganglionic synapses for sympathetic innervation to thoracic ganglia and to intra-visceral ganglia for parasympathetic efferent innervation, postganglionic fibers from sympathetic thoracic ganglia and neuroeffector junctions for parasympathetic mediated by acetylcholine and for sympathetic mediated by norepinephrine [3].

One of such cardiopulmonary inhibitory reflex is the BJR, thought to result from the stimulation of cardiopulmonary mechanoreceptors that are associated with unmyelinated vagal afferent nerve fibers, which constitute the afferent limb [7]. These chemo and mechanosensitive afferent C-fibers are composed of approximately $25 \%$ are myelinated fibers and $75 \%$ are nonmyelinated fibers whose receptors are located in the atria, ventricles, aorta, and lungs. Evidence suggests that the cardiac receptors mediating vasodepressor effects are preferentially distributed to the inferior wall of the heart [8]. These fibers terminate in the nucleus tractus solitaries (NTS) in the brain stem. The efferent component inhibits the sympathetic system and activates parasympathetic nervous system, resulting in bradycardia and peripheral vasodilation. Also, decreased activity of cardiac vagal afferent fibers enhances sympathetic activity and vascular resistance and promotes renin release and vasopressin secretion [1,9].

Stimulation of the C-fibers is thought to occur through mechanical or chemical means, which activates this reflex. Mechanical factors could be pressure, inotropism, volume changes whereas chemically stimulation is by the pharmacological agents such as veratrum alkaloids, nicotine, capsaicin, and a selective serotonergic 5-HT3 receptor agonist, phenyl biguanide (PBG), nitroglycerine etc. Rarely, radiographic contrast agents can induce the BJR, especially when injected into the right coronary artery [5].

The BJR is thought to activate cardiac receptors thus affecting developing pathophysiological responses or circulatory regulation. It occurs from peripheral venous pooling causing hypercontractility of the myocardium. This leads to reflex arterial vasodilatation and cardiac parasympathetic activation resulting in hypotension and bradycardia [10]. Activation of the cardiopulmonary vagal afferent fibers during the BJR blunts the efferent sympathetic nerve activity, thereby resulting in hypotension and bradycardia. Intracisternal or the NTS injection of the 5-HT3 receptor antagonist granisetron significantly attenuated the hypotension and bradycardia evoked by intravenous PBG, suggesting that the NTS is involved in the central pathway of the BJR [11].

Nitroglycerin has only rarely been implicated in eliciting a BJR. The mechanism is thought to be related to decrease in ventricular filling, which leads to a vigorous contraction of myocardial muscle fibers and the subsequent activation of ventricular mechanoreceptors that are innervated by C-fibers; this in turn evokes the BJR. BJR is inversely proportional to the intra sinus pressure of carotid sinus. The presence of tonic cardiovascular inhibitions exerted by the arterial baroreceptors tends to reduce the BJ reflex bradycardia and hypotension [12, 13]. Various clinical scenarios where BJR plays a role are discussed below.

\subsection{Blood Pressure Regulation}

The cardiac receptors mediating the BJR have very low basal firing rates in the absence of any stimulation. The output from these cardiac receptors contributes tonic inhibition to the vasomotor centers. These receptors, when stimulated by electrical excitation, show increased firing frequency with subsequent inhibition of the vasomotor centers, promoting vasodilation. BJR cardioinhibitory receptors, in conjunction 
with the aortic and carotid baroreceptors, participate in blood pressure regulation.

Decreased activity of the afferent limb of the BJR results in decreased firing of the efferent fibers of the BJR. This leads to reduced vasomotor inhibition with augmented medullary outflow and a consequent increase in blood pressure. However, with the baroreceptor reflex present, this increased blood pressure response is attenuated and there is no change in systemic blood pressure [6].

\subsection{Vasovagal Syncope}

The BJR overlaps with vasovagal syncope. The syndrome of cardiac slowing with hypotension or vasovagal syncope has been attributed to activation of the reflex. Factors such as pain, fear and reduced cardiac venous return disrupt the normal circulatory response and trigger the parasympathetic action and inhibit sympathetic activity and causes hypotension. This response may occur during regional anesthesia, hemorrhage or supine inferior vena cava compression in pregnancy; these factors are additive when combined [14].

Vasovagal syncope has been reported during shoulder arthroscopy in supine position, following cardiac procedures such as coronary angiography, injection of thrombolytic agents, and radio frequency catheter ablation of accessory pathway [3]. Many studies have attempted to tie such observations of bradycardia seen with hemorrhage to vasovagal mechanisms and therefore to the BJR. However, it remains unclear if this relationship truly exists [6].

\subsection{Hemorrhage and Hypovolemia}

The baroreceptor and cardioinhibitory receptor systems interact with one another during the maintenance of normal blood pressure and during hypovolemic insults. Acute bleeding is a major factor affecting perioperative circulatory dynamics. During mild hemorrhage, with baroreceptor-mediated sympatho-excitatory response, BP is well maintained by an increase in vascular resistance and heart rate. During moderate hypovolemia, when the central blood volume is critically reduced by more than $30 \%$, there is withdrawal of sympathetic drive and activation of parasympathetic drive. The BJR activity is withdrawn simultaneously with activation of the baroreceptor reflex leading to an increase in blood pressure.

During severe volume loss, when blood volume and cardiac filling become critically reduced, not only is the BJR, presumably paradoxically activated, but also the baroreceptor fails to exert its dominant blood pressure control effects either due to failed sensing at the afferent limb or inhibition of its efferent limb from some component of the BJR pathway. The net result is bradycardia and further hypotension [6]. The BJR is thought to be a protective response, defending the heart against ischemia during severe hypovolemia by reducing its workload through induction of bradycardia, negative inotropy, and afterload reduction [15].

\subsection{Myocardial Ischemia and Infarction}

Myocardial ischemia and infarction have been implicated several times as a sensitizing factor to the BJR. Physiologic significance of this reflex during myocardial ischemia depends on factors like interaction with arterial baroreflexes, engagement of various regional circulations by cardiac baroreflexes, distribution of ischemia and cardiac sensory receptors, and duration of the ischemia [9]. Mechanoreceptors as well as chemoreceptors might also be activated during myocardial ischemia. In cardiac diseases, there is increased serotonin level in the coronary circulation, whereas there is an increase in the myocardial acetylcholine level induced by intravenous PBG. Oxygen-derived free radicals and prostaglandins generated during ischemia and reperfusion also stimulate BJR. Stimulation of the BJR during myocardial ischemia produces particularly pronounced inhibition of renal sympathetic nerve traffic [5].

Excess activation of the BJR might cause severe bradycardia and hypotension, placing the patient's life at risk because the magnitude of sympathetic inhibition. Patients with infarction of the inferior wall of the left ventricle have been noted to have more frequent bradycardia and hypotension, resulting in sudden cardiac arrest presumed secondary to cardiac arrhythmia. The distribution of the cardioinhibitory $\mathrm{C}$-fiber afferent receptors in the myocardium correlates well with the coronary anatomy affected during myocardial ischemia and the concentration of cardiac inhibitory receptors greatest in the inferior and posterior walls of the heart. Some investigators have argued that the profound bradycardia and renal vasodilation reduces myocardial oxygen demand and augments renal perfusion that is beneficial in ischemia [8].

\subsection{Coronary Artery Stenosis and Aortic Stenosis}

Coronary stenosis alone, without concurrent ischemia could be a sensitizing factor for development of the BJR. As the afferent neural fiber bundle is anatomically near the left main coronary artery, a critical stenosis in this region may sensitize mechanoreceptors; however, the significance of this anatomic relationship as a predisposing factor for causing the BJR is uncertain and further investigations are recommended. Exertional syncope occurs in aortic stenosis secondary to reflex activation of the BJR. The increased left ventricular pressure trigger the cardioinhibitory reflexes and promote hypotension [7].

\subsection{Reperfusion-Induced Bezold-Jarisch Reflex}

BJR responses are observed in patients with inferior ischemia in association with coronary spasm and accounts for up to $61 \%$ brady arrhythmias occurring within the first hour of posterior (inferior) MI [9]. Research evidence suggests that locally released mediators that occur within minutes after the onset of ischemia, and particularly during reperfusion, such as prostaglandins, serotonin, and oxygen derived free radicals, may activate the cardiac vagal afferent pathways. Following the injection of intravenous thrombolysis with streptokinase, urokinase or rt-PA (accelerated tissue- type plasminogen activator) in acute MI patients, results in transient bradycardia 
and hypotension that is BJR [16].

Reperfusion stimulates the BJR, particularly in patients with inferior myocardial ischemia. As BJR response is indicative of successful reperfusion which occurs early after initiation of thrombolytic therapy, within the initial critical 2 hour window period of successful thrombolysis, therefore is of clinical significance. The appearance of the BJR enhances the probability of early reperfusion in up to $94 \%$. There is an added beneficial effect of this phenomenon in preserving sustained coronary patency. Hence, BJR in inferior acute myocardial infarction represents a reliable prognostic indicator of timely reperfusion and sustained coronary patency $[9,16]$.

\subsection{Coronary Arteriography}

There also exist evidence that the BJR may be involved in mediating the hypotension and bradycardia often seen during human coronary angiography with the injection of contrast media into the right coronary artery. Injection of contrast medium into the coronary arteries, supplying the inferior wall of the heart, results in bradycardia and hypotension more frequently than does injection into the artery supplying the anterior wall [8]. Depressor effects of coronary arteriography result from activation of coronary stretch receptors. It is believed that bradycardia is predominantly a reflex that originates mainly from sensory receptors in the inferior wall of the left ventricle. It also appears that the reflex is not stimulated primarily by the pressure of injection. Composition of the angiographic medium is more important than changes in coronary pressure for triggering the reflex [9].

\subsection{Anesthesia}

The most common side effects from spinal and epidural anesthesia are cardiovascular. In non-obstetrical population, the incidence of hypotension and bradycardia is $33 \%$ and $13 \%$ respectively. It is conceivable that the cardioinhibitory receptors of the BJR may be responsible for the bradycardia [6]. BJR is often incriminated as a cause of bradycardia and hypotension in various clinical settings such as stress testing and neuraxial anesthesia. However, its role in cases of neuraxial anesthesia induced cardiac arrest remains uncertain [12].

The postural change combined with the cardiovascular effects of the different anaesthetic drugs used, may trigger the occurrence of a cardiac arrest, due to a possible BJR. During rapid postural change, there is compression of the caudal vena cava by abdominal viscera, leading to a decreased venous return for a few seconds which stimulates the BJR. This may not be necessarily related to a hypovolemic state. Therefore it is advisable to be cautious of drugs affecting cardiovascular hemodynamics or sympatho-vagal balance as well as, to smoothly change the position of anaesthetized patient [13].

\subsection{Pregnancy}

Bezold Jarisch reflex plays a major role in explaining cardiovascular collapse resulting from diminished venous return during neuraxial interventions and in the supine inferior vena cava compression syndrome in pregnancy. BJR may promote diastolic filling during catastrophic declines in cardiac blood volume and may lead to asystole [1]. The reflex triggering this vagal response has been variably attributed to a mechanoreceptor response to fetal head compression, to baroreflex activation following increased blood pressure during umbilical cord compression, and /or a BJR response to reduced venous return from the placenta. Compression of the umbilical vein could reduce venous return from the placenta to the fetus sufficiently to reduce central blood volume. The reflex allows a longer filling time, restoring stroke volume and so restoring cardiac output [17].

\subsection{Testosterone and BJR}

The hormone testosterone influences renin angiotensin system which further influences BJR. The cardiopulmonary reflexes that are sensitive to chemical stimulation are also activated by bradykinin and degraded by angiotensin converting enzyme (ACE). Once ACE activity is reduced, an elevation of bradykinin occurs which desensitizes 4.10 . the 5-HT3 receptors in the vagal afferent pathways and impairs BJR. The castration results in a reduction in plasmatic levels of testosterone and a decrease in plasmatic and cardiac ACE activity without influencing $17 \beta$-estradiol levels and cardiac morphological parameters and thus could result in the impairment of the BJR [4].

\subsection{Cushing's Syndrome}

Clinical occurrence of BJR in patients with Cushing's syndrome is rare due to its sodium retention and volume expansion. During surgical interventions in these patients, the impact of body position and the applied weight force on the inferior vena cava (IVC) can have an effect on the central venous return. As IVC runs along the right side of the aorta and is located slightly to the right of lumbar vertebral bodies, is more compressed in the right lateral position than in the supine position which stimulates the receptors thus resulting in BJR [2].

\subsection{Laughter Induced Syncope}

BJR has been proposed to explain the mechanism behind laughter-induced syncope. Intense laughter causes episodes of prolonged forced expiration against a closed glottis, resulting in decreased lung volume and elevated intrathoracic pressure. The increase in intrathoracic pressure, decreases venous return to the heart, activating baroreceptors that in turn reduce sympathetic activity and heightens parasympathetic cardiovascular tone. These lead to hypotension and bradycardia that result in sudden cerebrovascular hypo perfusion and subsequent loss of consciousness [18].

\subsection{Neurosurgery: Craniotomy and Spinal Surgeries}

Although simultaneous occurrence of bradycardia and hypotension is rare, compared to other cardiovascular changes during craniotomy but are not uncommon. During craniotomy the sudden fall in the elevated intracranial pressure (ICP) 
following bone flap removal decreases the systemic vascular resistance aggravating the reduction in intracardiac volume. Along with these, mannitol which is used as an osmotic agent, induces diuresis which reduces intravascular volume and the upright position $\left(45^{\circ}\right)$ during the surgery produces peripheral venous pooling, thus reducing the venous return to the heart. These factors stimulate the pressure receptors in the under filled ventricular wall and trigger activation of the afferent $\mathrm{c}^{-}$ fibres and thus the BJR [10].

The causes of intraoperative junctional rhythm include surgical stress response, vagal hypertonicity, hypothermia, endotracheal tube cuff pressure or venous air embolism. The use of prone position may result in decreased venous return from pooling of blood in the legs and decreased left ventricular compliance secondary to increased intrathoracic pressure for spine surgery, causing a decrease in both arterial pressure and tissue perfusion $[19,20]$.

\subsection{Diabetes}

Earlier it was believed that as both the sympathetic and parasympathetic nervous system are impaired in Diabetic patients, there is impaired BJR. On the contrary, recent studies have suggested Diabetes impairs the arterial baroreflex, but not the BJR. Diabetic neuropathy is involved in enhancement of the BJR. The cardio depressor response due to the enhanced BJR may cause autonomic disturbance in patients with diabetic autonomic neuropathy leading to severe bradycardia and hypotension, placing the patient's life at risk. Maintaining cardiac filling with the administration of dextran is proved to inhibit the BJR [15].

\subsection{Bezold-Jarisch Reflex as a Defense Mechanism Against Toxic Chemical Hazards}

The chemical inhalants activate the cardiac component of the BJR and results in asystole and bradycardia. This chemoreflex response retards systemic distribution of the absorbed chemical pollutant, after escaping constriction of pulmonary veins that are more reactive than the proximal arterioles [3].

\section{Conclusion}

Simultaneous occurrence of bradycardia and hypotension should alert clinicians to the possibility of BJR. Once BJR occurs, aggressive fluid resuscitation and administration of vagolytic such as atropine and if unresponsive, use of vasopressors should be considered.

\section{Recommendation}

Despite the appealing theoretic relation between BJR and the above-mentioned clinical scenarios, further laboratory and clinical investigation should be undertaken. Stimulators and inhibitors of the cardio-inhibitory receptors in humans need to be characterized and the full spectrum of the human efferent response to these factors should be investigated.

\section{Acknowledgements}

Humble gratitude to Dr A. K Singh (Medical advisor) and Dr Sandeep Tanwar (G. M) for providing resources and constant encouragement towards this review article's completion.

Special thanks to Maj. Rahul Prashad (Medical Superintendent) and Dr Ravikant Gupta (Medical Director), Max Super Speciality Hospital, Dehradun, for their advice on manuscript preparation, guidance and valuable inputs.

\section{References}

[1] Conley A, Biddle C, Baker K. A tour of autonomic reflex activity relevant to clinical practice. AANA J. 2017; 85 (2): $141-7$

[2] Kim YH, Kim DJ, Kim WY. Bezold-Jarisch reflex caused by postural change. J Anesth 2015; 29 (1): 158.

[3] Aviado DM, Aviado DG. The Bezold-Jarisch reflex. A historical perspective of cardiopulmonary reflexes. Ann N Y Acad Sci. 2001; 940: 48-58.

[4] Heringer OA, Cassaro KOD, Mateus, NC et al. Relationship between male hormonal status, Bezold-Jarisch reflex function and ACE activity (cardiac and plasmatic). Can J PhysiolPharmacol. 2016; 94 (2): 231-6.

[5] Kashihara K. Roles of arterial baroreceptor reflex during Bezold-Jarisch Reflex. CurrCardiol Rev. 2009; 5 (4): 263-7.

[6] Campagna JA, Carter C. Clinical relevance of the BezoldJarisch reflex. Anesthesiology. 2003; 98 (5): 1250-60.

[7] Shah S, Waxman S. Two cases of Bezold-Jarisch reflex induced by intra-arterial nitroglycerin in critical left main coronary artery stenosis. Tex Heart Inst J. 2013; 40 (4): 484-6.

[8] Thames MD, Klopfenstein HS, Abboud FM, Mark AL and Walker JL. Preferential distribution of inhibitory cardiac receptors with vagal afferents to the inferoposterior wall of the left ventricle activated during coronary occlusion in the dog. Circ Res. 1978; 43 (4): 512-9.

[9] Mark AL. The Bezold-Jarischreflex revisited: clinical implications of inhibitory reflexes originating in the heart. J Am CollCardiol. 1983; 1 (1): 90-102.

[10] Bilgi K, Kamath S, Sultana N. BezoldJarisch reflex and acute cardiovascular collapse during craniotomy. Indian J Anaesth. 2017; 61 (2): 176-7.

[11] Pires JG, Silva SR, Ramage AG, Futuro-Neto HA. Evidence that 5-HT3 receptors in the nucleus tractussolitarius and other brainstem areas modulate the vagal bradycardia evoked by activation of the von Bezold-Jarisch reflex in the anesthetized rat. Brain Res. 1998; 791 (1-2): 229-34.

[12] Parent ME, Lepage S. A heart stopping case of theBezold-Jarischreflex. Case Rep Cardiol. 2015; 2015: 359401. http://dx.doi.org/10.1155/2015/359401.

[13] Conde Ruiz C, Junot S. Successful cardiopulmonary resuscitation in a sevoflurane anesthetized horse that suffered cardiac arrest at recovery. Front Vet Sci. 2018; 5: 138. doi: $10.3389 /$ fvets.2018.00138. 
[14] Kinsella SM, Tuckey JP. Perioperative bradycardia and asystole: relationship to vasovagal syncope and the BezoldJarisch reflex. Br J Anaesth 2001; 86 (6): 859-68.

[15] Boku A, Sugimura M, Morimoto Y, Hanamoto H, Niwa H. Hemodynamic and autonomic response to acute hemorrhage in streptozotocin-induced diabetic rats. CardiovascDiabetol. 2010; 9: 78.

[16] John A. Chiladakis JA, Patsouras N, Manolis AS. The Bezold-Jarisch Reflex in acute inferior myocardial infarction: clinical and sympathovagal spectral correlates. Clin. Cardiol. 2003; 26 : 323-8.

[17] Lear CA, GalinskyR, Wassink G, et al. The myths and physiology surrounding intrapartum decelerations: the critical role of the peripheral chemoreflex. J Physiol. 2016; 594 (17): $4711-25$.

[18] Biso S, Wongrakpanich S, Agrawal A, et al. Laughter-induced syncope: literature review. J Cardiovasc Disease Res. 2017; 8 (3): 66-71.

[19] Agrawal S, Srivastava V. BezoldJarisch reflex as a cause of haemodynamic alterations during surgery in prone position. Anaesth, Pain \& Intensive Care. 2010; 14 (2): 115-7.

[20] Guerri-Guttenberg RA, Siaba-Serrate F, CacheiroFJ. Clinical relevance of cardiopulmonary reflexes in anesthesiology Rev EspAnestesiolReanim. 2013; 60 (8): 448-56. 